\title{
A Facile Reaction Strategy for the Synthesis of MOF-based Pine Needles-like Nanoclusters Hierarchical Structure for Efficient \\ Overall Water Splitting
}

Zhenqi Huang ${ }^{\mathrm{a}}$, Lei Hao ${ }^{\mathrm{a}}$, Xinxing Ma ${ }^{\mathrm{a}}$, Shihui Zhang ${ }^{\mathrm{a}}$, Run Zhang ${ }^{\mathrm{a}}$, Kefen Yue ${ }^{\mathrm{a}^{*} \text {, }}$ Yaoyu Wang ${ }^{\mathrm{a}}$

${ }^{\text {a }}$ College of Chemistry and Materials Science, Key Laboratory of

Synthetic and Natural Functional Molecule of the Ministry of Education, National Demonstration Center for Experimental Chemistry Education, Shaanxi Key Laboratory of Physico-Inorganic Chemistry, Northwest University, No. 1, Xuefu Ave., Xi'an, 710127, China.

${ }^{*}$ Corresponding authors. E-mail: ykflyy@nwu.edu.cn 
Number of Pages: 23

Number of Figures: 13

Number of Tables: 6

\section{Content}

Fig. S1. The connection modes of complex deprotonated bta ${ }^{4-}$ linkers.

Fig. S2. The SEM images of Nickel Foam and $\mathrm{Co}(\mathrm{OH}) \mathrm{F} / \mathrm{NF}$ (a) Nickel Foam, (b) low-magnification and (c) high-magnification of $\mathrm{Co}(\mathrm{OH}) \mathrm{F} / \mathrm{NF}$.

Fig. S3. EDX spectrum and corresponding elemental compositions of the $\mathrm{Co}_{0.4}(\mathrm{Hatz})_{0.1}\left(\mathrm{H}_{4} \mathrm{bta}\right)_{0.1} / \mathrm{NF}$.

Fig. S4. The SEM images of $\mathrm{Co}_{x}(\mathrm{Hatz})_{0.1}\left(\mathrm{H}_{4} \mathrm{bta}\right)_{0.1} / \mathrm{NF}(x=0.1,0.2,0.4$, and 0.5$)$ : lowmagnification (left) and high-magnification (right): (a) and (b) for $\mathrm{Co}_{0.1}(\mathrm{Hatz})_{0.1}\left(\mathrm{H}_{4} \mathrm{bta}\right)_{0.1} / \mathrm{NF}$, (c) and (d) for $\mathrm{Co}_{0.2}(\mathrm{Hatz})_{0.1}\left(\mathrm{H}_{4} \mathrm{bta}\right)_{0.1} / \mathrm{NF}$, (e) and (f) for $\mathrm{Co}_{0.4}(\mathrm{Hatz})_{0.1}\left(\mathrm{H}_{4} \mathrm{bta}\right)_{0.1} / \mathrm{NF},(\mathrm{g})$ and $(\mathrm{h})$ for $\mathrm{Co}_{0.5}(\mathrm{Hatz})_{0.1}\left(\mathrm{H}_{4} \mathrm{bta}\right)_{0.1} / \mathrm{NF}$, respectively.

Fig. S5. The XRD patterns of $\mathrm{Co}(\mathrm{OH}) \mathrm{F} / \mathrm{NF}$.

Fig. S6. The FT-IR spectroscopy of $\mathrm{Co}_{0.5}(\mathrm{Hatz})_{0.1}\left(\mathrm{H}_{4} \mathrm{bta}\right)_{0.1} / \mathrm{NF}$ and $\mathrm{Co}(\mathrm{OH}) \mathrm{F} / \mathrm{NF}$.

Fig. S7. High-resolution XPS spectra of Co $2 p$ in (a) $\mathrm{Co}_{0.1}(\mathrm{Hatz})_{0.1}\left(\mathrm{H}_{4} \mathrm{bta}\right)_{0.1} / \mathrm{NF}$, (b) $\mathrm{Co}_{0.2}(\mathrm{Hatz})_{0.1}\left(\mathrm{H}_{4} \mathrm{bta}\right)_{0.1} / \mathrm{NF},(\mathrm{c}) \mathrm{Co}_{0.4}(\mathrm{Hatz})_{0.1}\left(\mathrm{H}_{4} \mathrm{bta}\right)_{0.1} / \mathrm{NF}$, and (d)

$\mathrm{Co}_{0.5}(\mathrm{Hatz})_{0.1}\left(\mathrm{H}_{4} \mathrm{bta}\right)_{0.1} / \mathrm{NF}$, respectively.

Fig. S8. (a) The double layer capacitances $\left(C_{\mathrm{dl}}\right)$ of $\mathrm{Co}_{x}(\mathrm{Hatz})_{0.1}\left(\mathrm{H}_{4} \mathrm{bta}\right)_{0.1} / \mathrm{NF}(x=0.1$, $0.2,0.4$, and 0.5 ) and NF at the potential of $0.9 \mathrm{~V}$ vs. RHE and the CV curves at various scan rates $\left(20,40,60,80,100 \mathrm{mV} \mathrm{s}^{-1}\right)$ in the potential range $0.8-1.0 \mathrm{~V}$ vs.

RHE for (b) $\mathrm{Co}_{0.1}(\mathrm{Hatz})_{0.1}\left(\mathrm{H}_{4} \mathrm{bta}\right)_{0.1} / \mathrm{NF}$, (c) $\mathrm{Co}_{0.2}(\mathrm{Hatz})_{0.1}\left(\mathrm{H}_{4} \mathrm{bta}\right)_{0.1} / \mathrm{NF}$, (d)

$\mathrm{Co}_{0.4}(\mathrm{Hatz})_{0.1}\left(\mathrm{H}_{4} \mathrm{bta}\right)_{0.1} / \mathrm{NF}$, (e) $\mathrm{Co}_{0.5}(\mathrm{Hatz})_{0.1}\left(\mathrm{H}_{4} \mathrm{bta}\right)_{0.1} / \mathrm{NF}$, and (f) NF.

Fig. S9. Electrocatalytic OER performance of $\mathrm{Co}_{0.4}(\mathrm{Hatz})_{0.1}\left(\mathrm{H}_{4} \mathrm{bta}\right)_{0.1} / \mathrm{NF}$ and crystalline powder coated on the NF in $1.0 \mathrm{M} \mathrm{KOH}$ : (a) LSV polarization curves, (b) EIS of $\mathrm{Co}_{0.4}(\mathrm{Hatz})_{0.1}\left(\mathrm{H}_{4} \mathrm{bta}\right)_{0.1} / \mathrm{NF}$ and crystalline powder coated on the NF, (c) the $\mathrm{CV}$ curves at various scan rates in the potential range $0.8-1.0 \mathrm{~V}$ vs. RHE for 
crystalline powder coated on the NF, (d) the Cdl of $\mathrm{Co}_{0.4}(\mathrm{Hatz})_{0.1}\left(\mathrm{H}_{4} \mathrm{bta}\right)_{0.1} / \mathrm{NF}$ and crystalline powder coated on the NF at the potential of $0.9 \mathrm{~V}$ vs. RHE.

Fig. S10. The EDX spectrum and corresponding elemental compositions of $\mathrm{Co}_{0.4}(\mathrm{Hatz})_{0.1}\left(\mathrm{H}_{4} \mathrm{bta}\right)_{0.1} / \mathrm{NF}$ after OER stability test.

Fig. S11. High-resolution XPS spectra of $\mathrm{Co}_{0.4}(\mathrm{Hatz})_{0.1}\left(\mathrm{H}_{4} \mathrm{bta}\right)_{0.1} / \mathrm{NF}$ after OER stability test: (a) C 1s, (b) O 1s, (c) Co 2p and (d) Ni 2p.

Fig. S12. Electrocatalytic HER performance of $\mathrm{Co}_{0.4}(\mathrm{Hatz})_{0.1}\left(\mathrm{H}_{4} \mathrm{bta}\right)_{0.1} / \mathrm{NF}$ and crystalline powder coated on the NF in $1.0 \mathrm{M} \mathrm{KOH}$ : (a) LSV polarization curves, (b) EIS of $\mathrm{Co}_{0.4}(\mathrm{Hatz})_{0.1}\left(\mathrm{H}_{4} \mathrm{bta}\right)_{0.1} / \mathrm{NF}$ and crystalline powder coated on the NF.

Fig. S13. (a) The digital photo of the experimental equipment, (b) The digital photo of $\mathrm{H}_{2}$ and $\mathrm{O}_{2}$ volume at different test time, (c) Volume of $\mathrm{H}_{2}$ and $\mathrm{O}_{2}$ as a function time at $0.025 \mathrm{~A}$.

Table S1. The crystallographic date and structure refinement results of $\left\{\left[\mathrm{Co}_{2}(\mathrm{Hatz})(\mathrm{bta})\right] \cdot \mathrm{H}_{2} \mathrm{O}\right\}$ n.

Table S2. The selected bond lengths and angles of $\left\{\left[\mathrm{Co}_{2}(\mathrm{Hatz})(\mathrm{bta})\right] \cdot \mathrm{H}_{2} \mathrm{O}\right\}_{\mathrm{n}}$.

Table S3. The OER catalytic properties for different samples.

Table S4. EIS characterization of various electrodes. Values of the main parameters of equivalent circuit elements.

Table S5. The HER catalytic properties for different samples.

Table S6. The comparison for overall water electrolysis activities for various catalysts. 
a

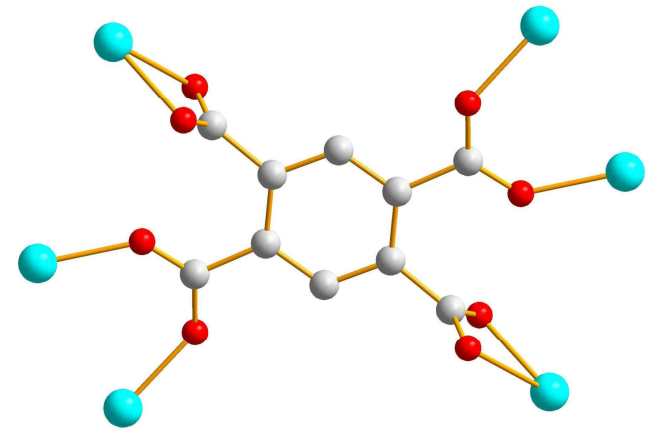

b

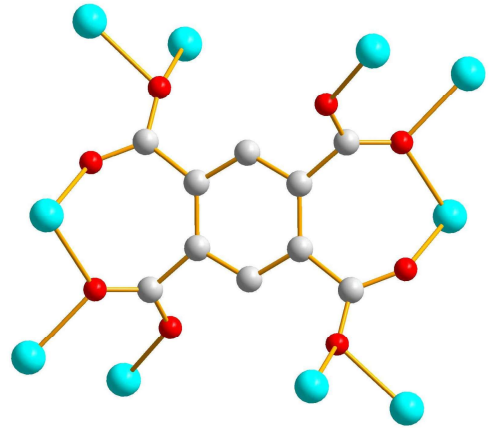

Fig. S1. The connection modes of complex deprotonated bta ${ }^{4-}$ linkers. 

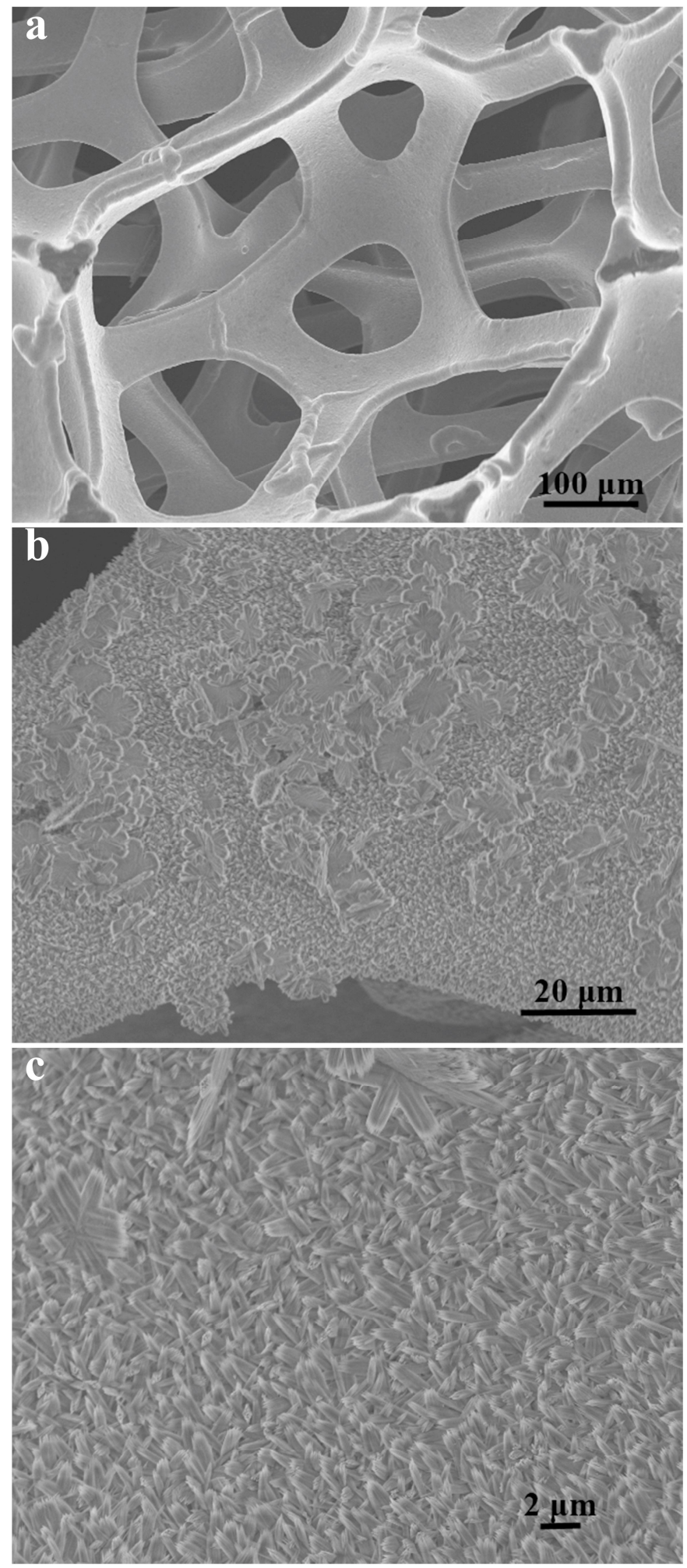

Fig. S2. The SEM images of Nickel Foam and $\mathrm{Co}(\mathrm{OH}) \mathrm{F} / \mathrm{NF}$ (a) Nickel Foam, (b) low-magnification and (c) high-magnification of $\mathrm{Co}(\mathrm{OH}) \mathrm{F} / \mathrm{NF}$. 


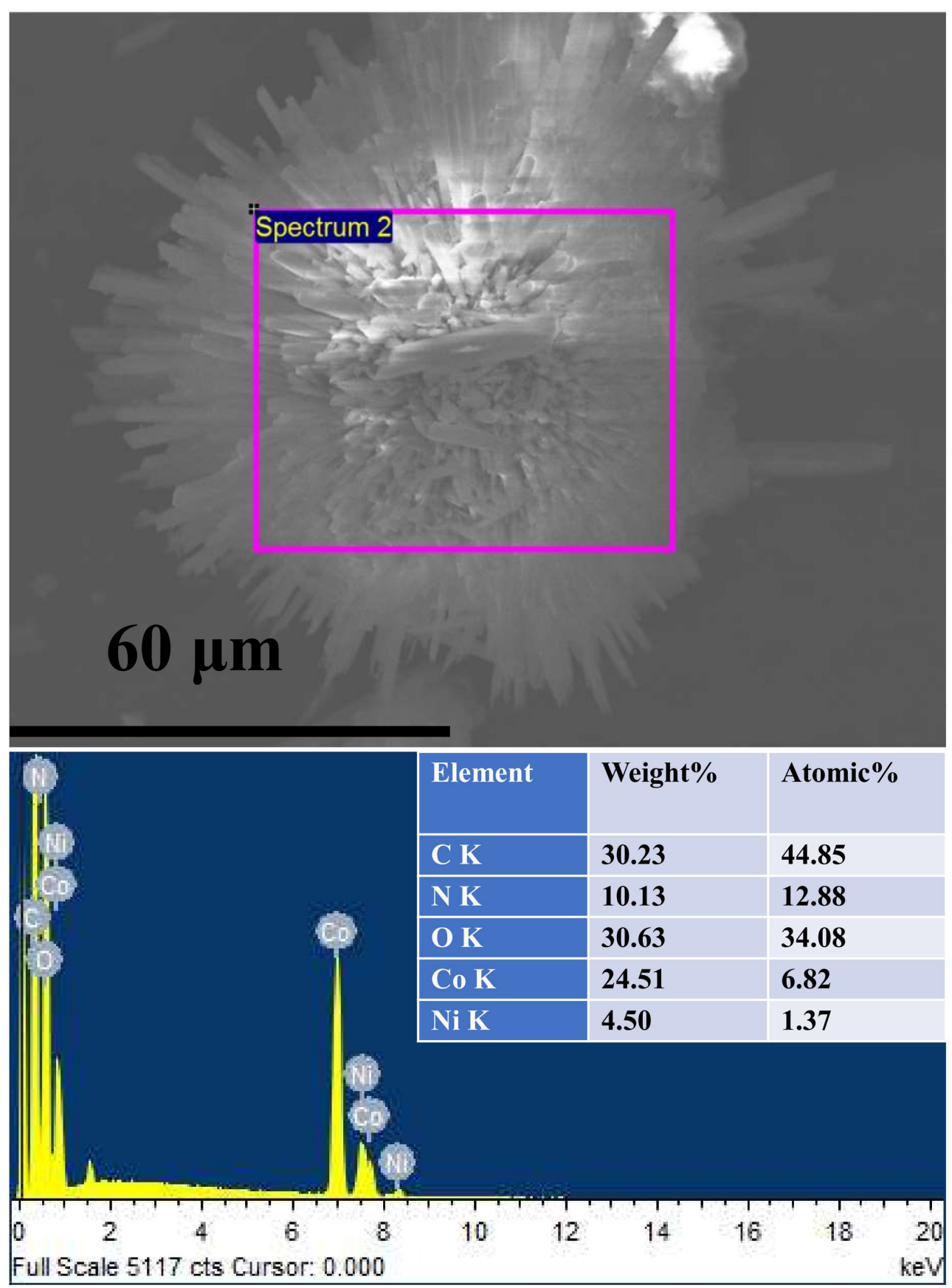

Fig. S3. EDX spectrum and corresponding elemental compositions of the $\mathrm{Co}_{0.4}(\mathrm{Hatz})_{0.1}\left(\mathrm{H}_{4} \mathrm{bta}\right)_{0.1} / \mathrm{NF}$. 


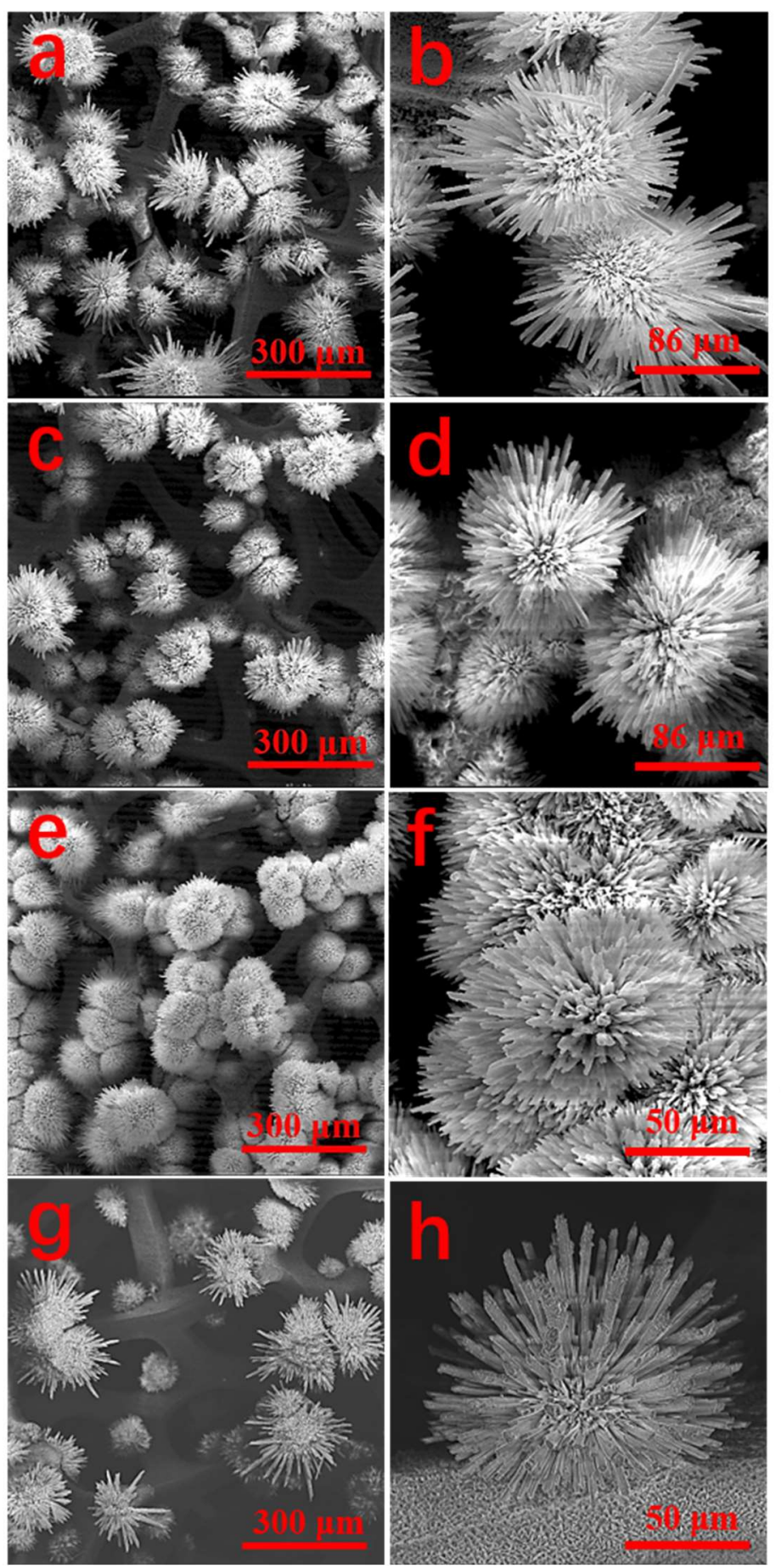

Fig. S4. The SEM images of $\mathrm{Co}_{x}(\mathrm{Hatz})_{0.1}\left(\mathrm{H}_{4} \mathrm{bta}\right)_{0.1} / \mathrm{NF}(x=0.1,0.2,0.4$, and 0.5$)$ : lowmagnification (left) and high-magnification (right): (a) and (b) for $\mathrm{Co}_{0.1}(\mathrm{Hatz})_{0.1}\left(\mathrm{H}_{4} \mathrm{bta}\right)_{0.1} / \mathrm{NF}$, (c) and (d) for $\mathrm{Co}_{0.2}(\mathrm{Hatz})_{0.1}\left(\mathrm{H}_{4} \mathrm{bta}\right)_{0.1} / \mathrm{NF}$, (e) and (f) for $\mathrm{Co}_{0.4}(\mathrm{Hatz})_{0.1}\left(\mathrm{H}_{4} \mathrm{bta}\right)_{0.1} / \mathrm{NF}$, (g) and (h) for $\mathrm{Co}_{0.5}(\mathrm{Hatz})_{0.1}\left(\mathrm{H}_{4} \mathrm{bta}\right)_{0.1} / \mathrm{NF}$, respectively 


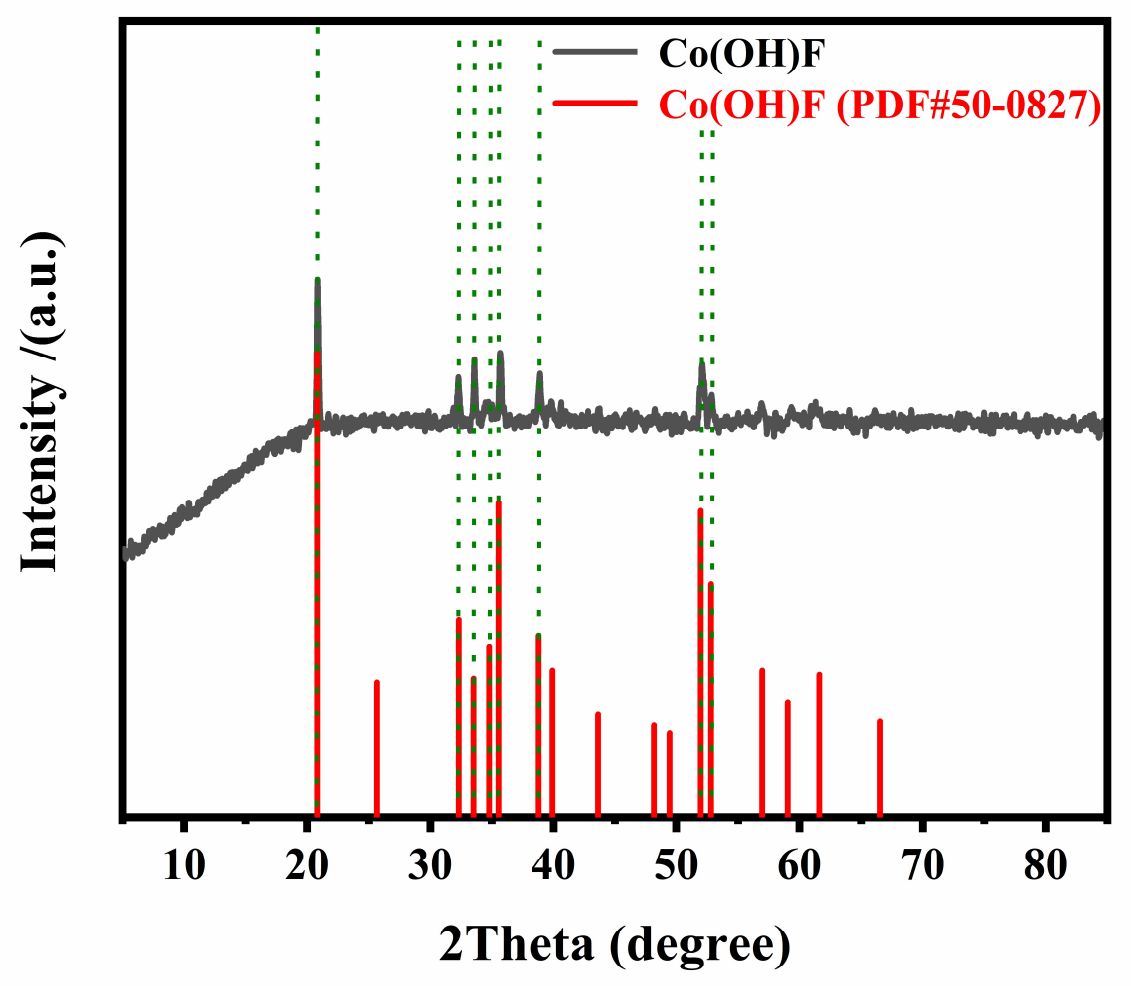

Fig. S5. The XRD patterns of $\mathrm{Co}(\mathrm{OH}) \mathrm{F} / \mathrm{NF}$. 


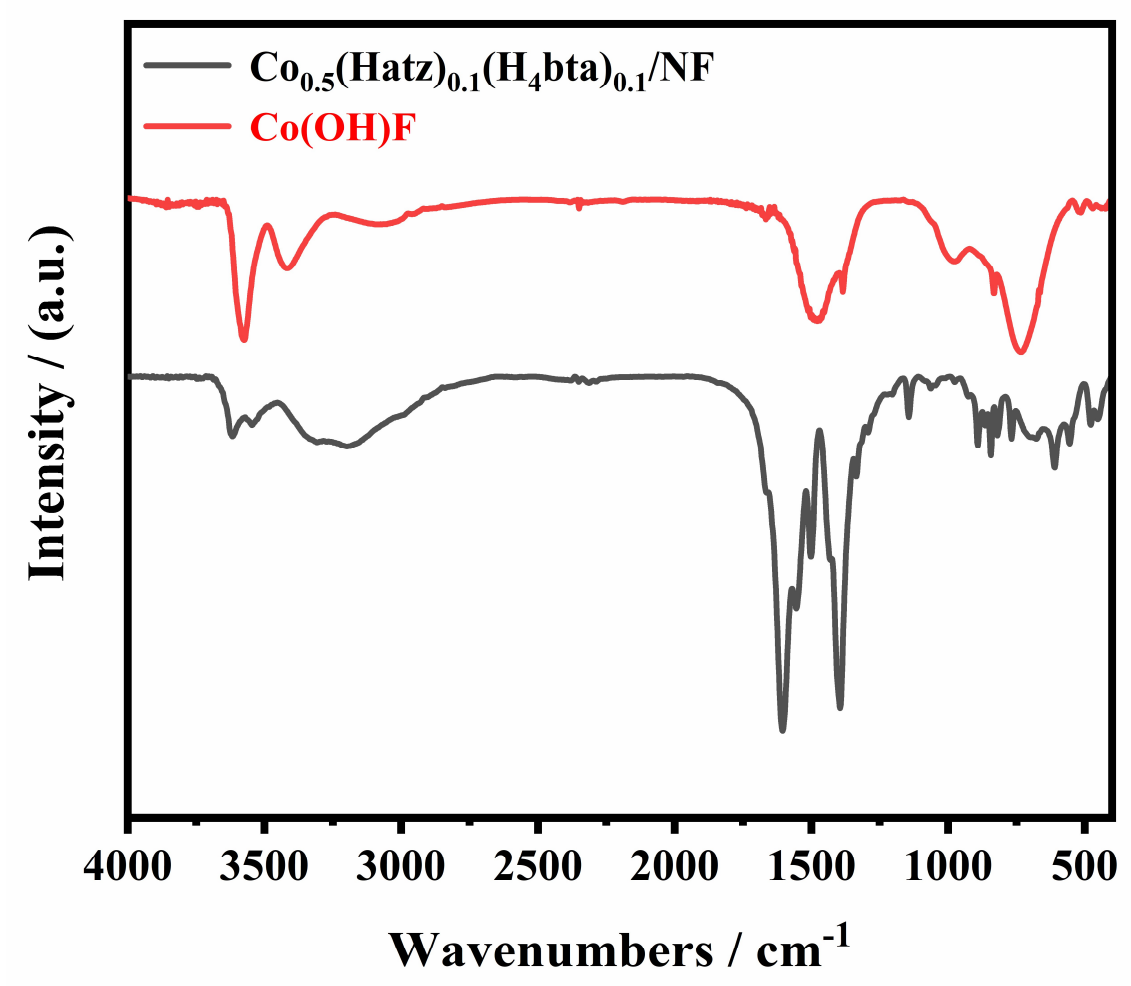

Fig. S6. The FT-IR spectroscopy of $\mathrm{Co}_{0.5}(\mathrm{Hatz})_{0.1}\left(\mathrm{H}_{4} \mathrm{bta}\right)_{0.1} / \mathrm{NF}$ and $\mathrm{Co}(\mathrm{OH}) \mathrm{F} / \mathrm{NF}$. 

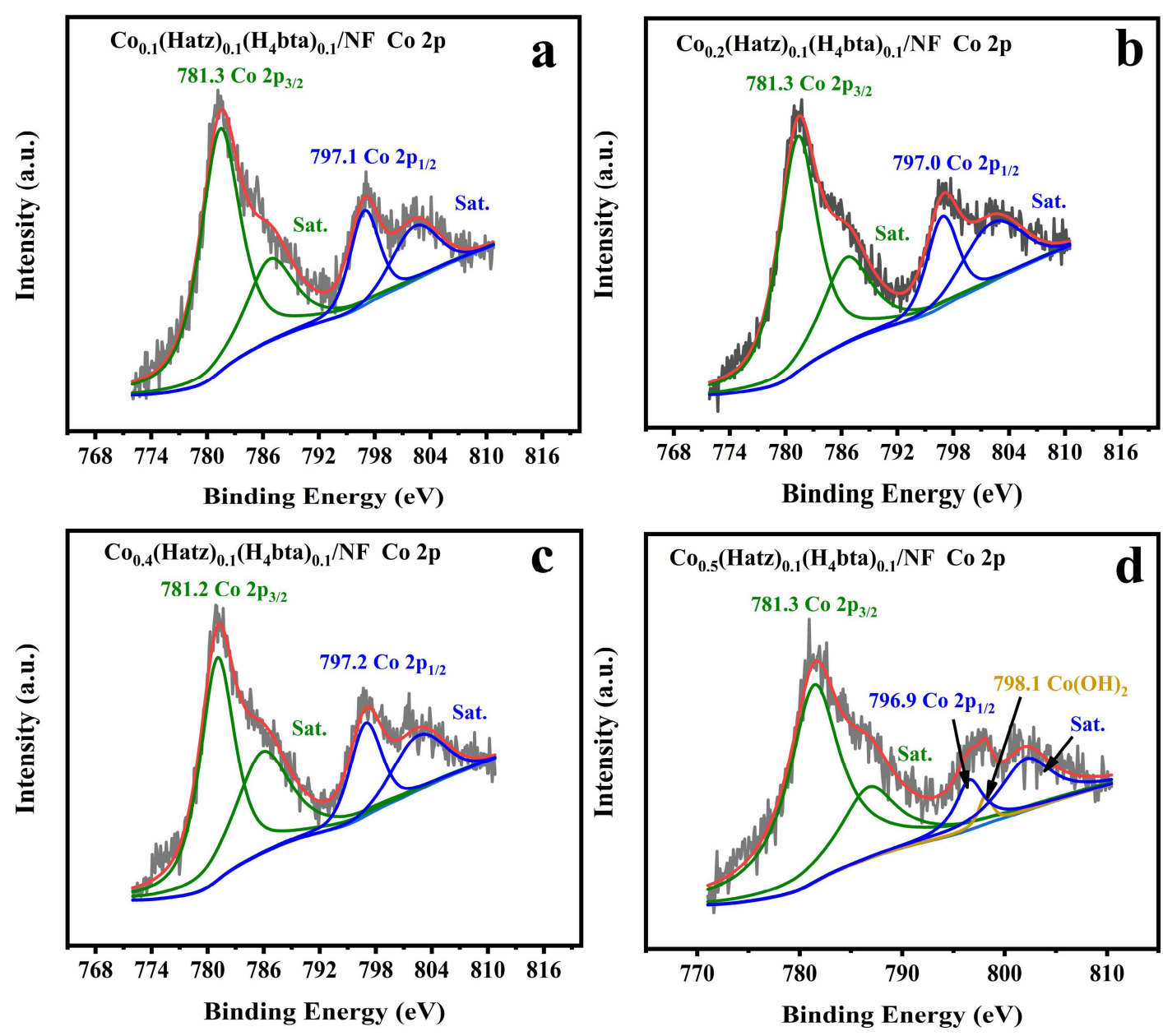

Fig. S7. High-resolution XPS spectra of Co $2 p$ in (a) $\mathrm{Co}_{0.1}(\mathrm{Hatz})_{0.1}\left(\mathrm{H}_{4} \mathrm{bta}\right)_{0.1} / \mathrm{NF}$, (b) $\mathrm{Co}_{0.2}(\mathrm{Hatz})_{0.1}\left(\mathrm{H}_{4} \mathrm{bta}\right)_{0.1} / \mathrm{NF}$, (c) $\mathrm{Co}_{0.4}(\mathrm{Hatz})_{0.1}\left(\mathrm{H}_{4} \mathrm{bta}\right)_{0.1} / \mathrm{NF}$, and (d) $\mathrm{Co}_{0.5}(\mathrm{Hatz})_{0.1}\left(\mathrm{H}_{4} \mathrm{bta}\right)_{0.1} / \mathrm{NF}$, respectively. 

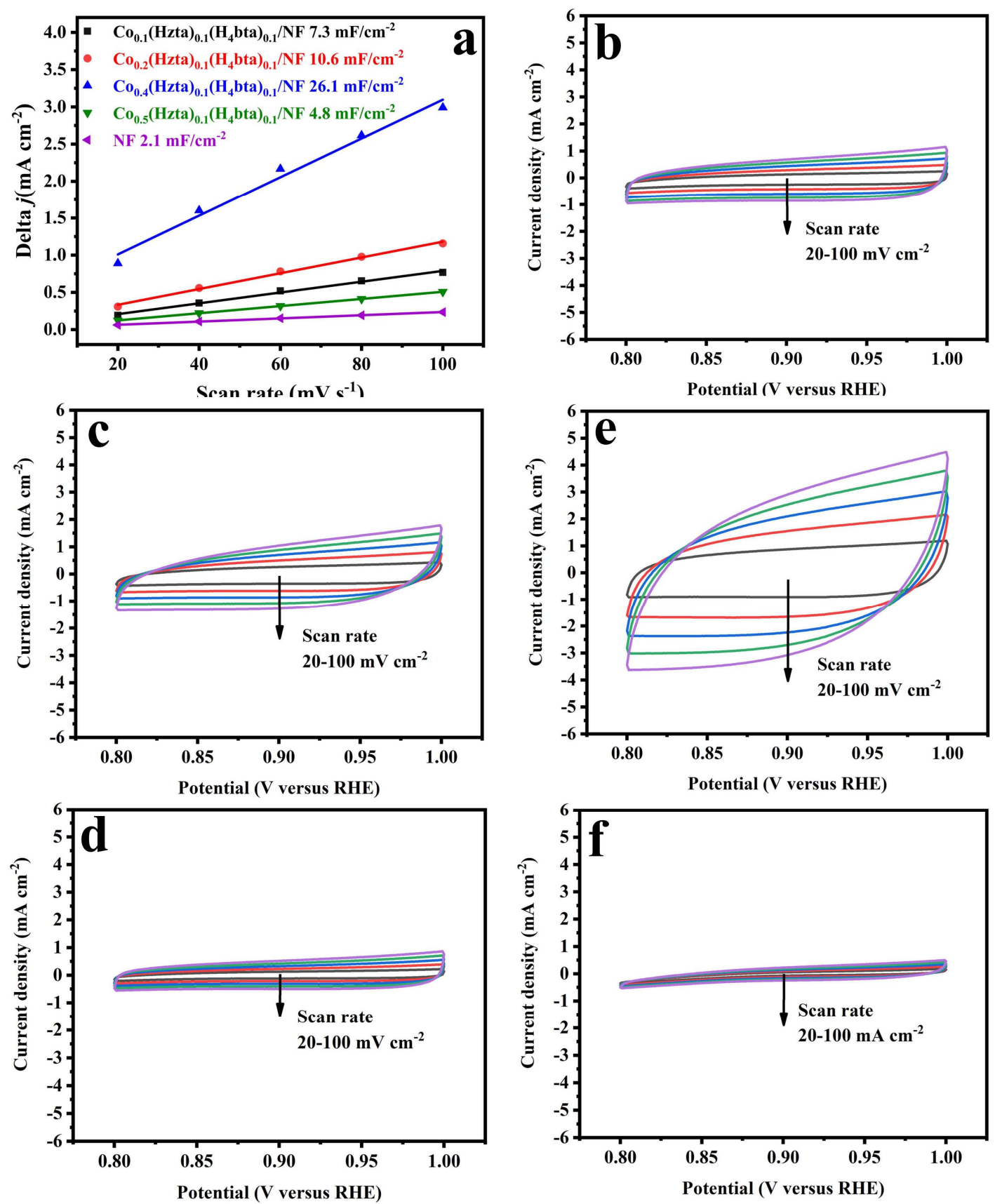

Fig. S8. (a) The double layer capacitances $\left(C_{\mathrm{dl}}\right)$ of $\mathrm{Co}_{x}(\mathrm{Hatz})_{0.1}\left(\mathrm{H}_{4} \mathrm{bta}\right)_{0.1} / \mathrm{NF}(x=0.1$, $0.2,0.4$, and 0.5 ) and NF at the potential of $0.9 \mathrm{~V}$ vs. RHE and the CV curves at various scan rates $\left(20,40,60,80,100 \mathrm{mV} \mathrm{s}^{-1}\right)$ in the potential range $0.8-1.0 \mathrm{~V}$ vs.

RHE for (b) $\mathrm{Co}_{0.1}(\mathrm{Hatz})_{0.1}\left(\mathrm{H}_{4} \mathrm{bta}\right)_{0.1} / \mathrm{NF}$, (c) $\mathrm{Co}_{0.2}(\mathrm{Hatz})_{0.1}\left(\mathrm{H}_{4} \mathrm{bta}\right)_{0.1} / \mathrm{NF}$, (d) $\mathrm{Co}_{0.4}(\mathrm{Hatz})_{0.1}\left(\mathrm{H}_{4} \mathrm{bta}\right)_{0.1} / \mathrm{NF}$, (e) $\mathrm{Co}_{0.5}(\mathrm{Hatz})_{0.1}\left(\mathrm{H}_{4} \mathrm{bta}\right)_{0.1} / \mathrm{NF}$, and (f) NF. 

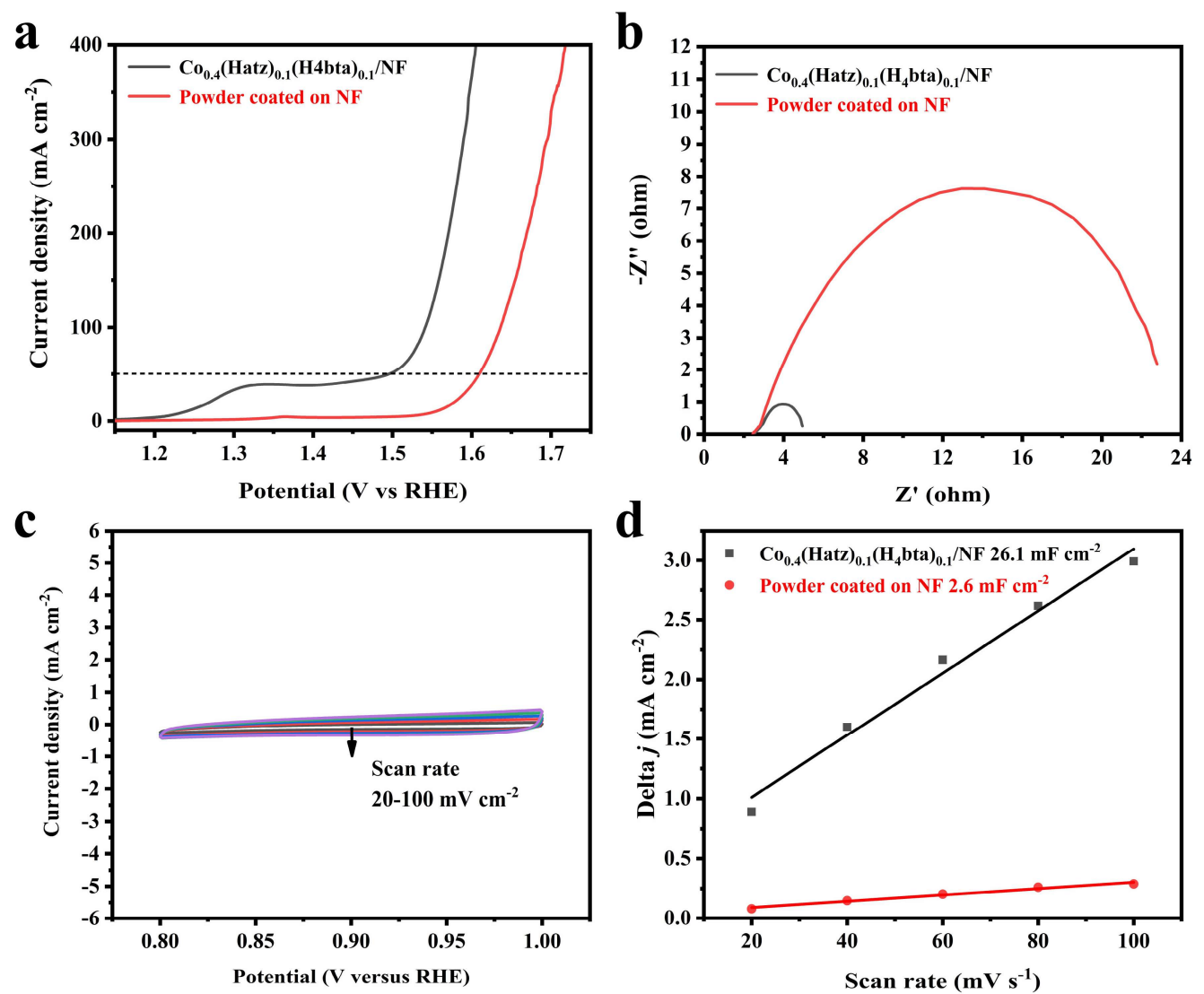

Fig. S9. Electrocatalytic OER performance of $\mathrm{Co}_{0.4}(\mathrm{Hatz})_{0.1}\left(\mathrm{H}_{4} \mathrm{bta}\right)_{0.1} / \mathrm{NF}$ and crystalline powder coated on the NF in 1.0 M KOH: (a) LSV polarization curves, (b) EIS of $\mathrm{Co}_{0.4}(\mathrm{Hatz})_{0.1}\left(\mathrm{H}_{4} \mathrm{bta}\right)_{0.1} / \mathrm{NF}$ and crystalline powder coated on the NF, (c) the

$\mathrm{CV}$ curves at various scan rates in the potential range $0.8-1.0 \mathrm{~V}$ vs. RHE for crystalline powder coated on the NF, (d) the $\mathrm{Cdl}$ of $\mathrm{Co}_{0.4}(\mathrm{Hatz})_{0.1}\left(\mathrm{H}_{4} \mathrm{bta}\right)_{0.1} / \mathrm{NF}$ and crystalline powder coated on the NF at the potential of $0.9 \mathrm{~V}$ vs. RHE. 


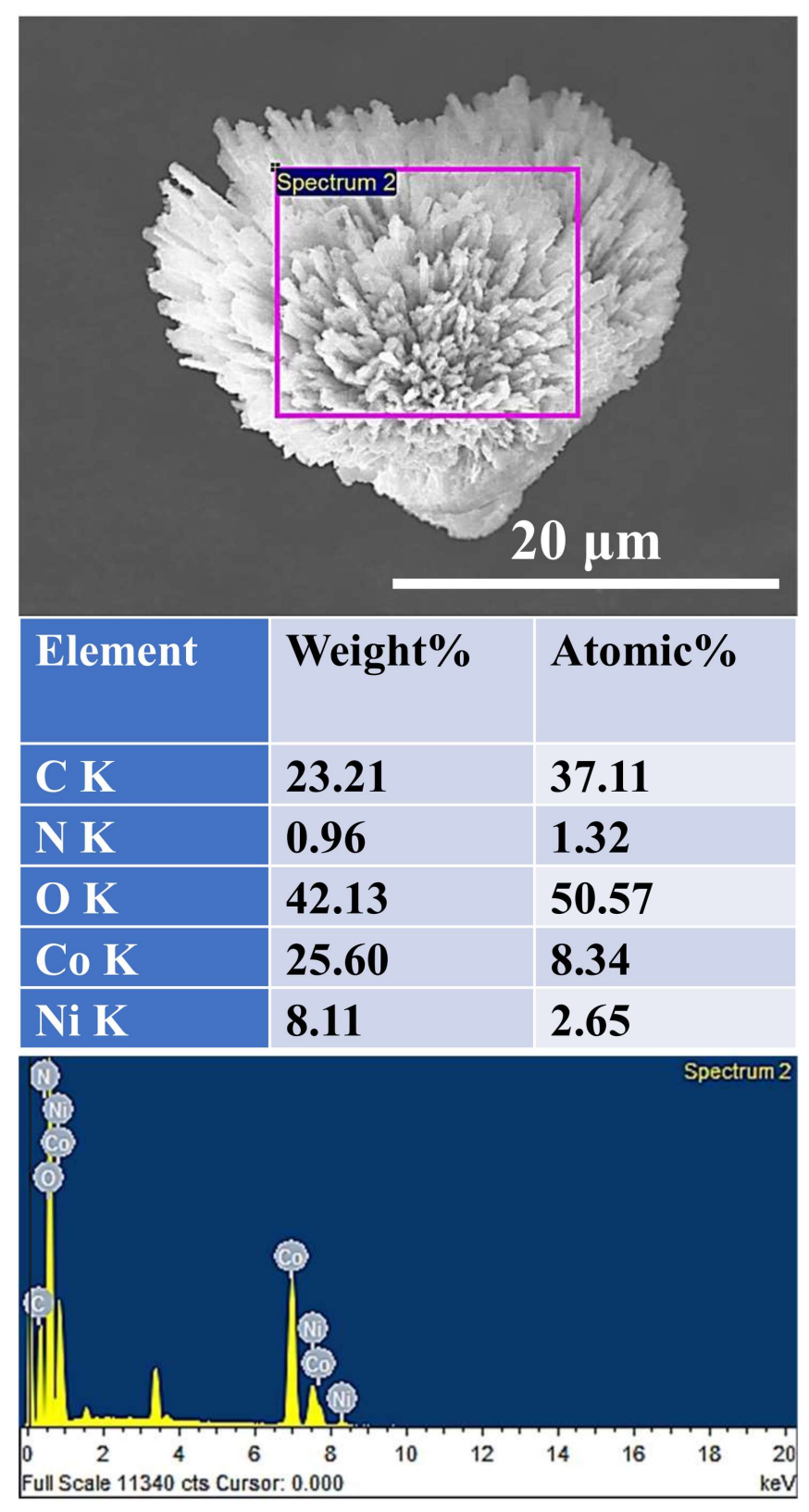

Fig. S10. The EDX spectrum and corresponding elemental compositions of $\mathrm{Co}_{0.4}(\mathrm{Hatz})_{0.1}\left(\mathrm{H}_{4} \mathrm{bta}\right)_{0.1} / \mathrm{NF}$ after OER stability test. 

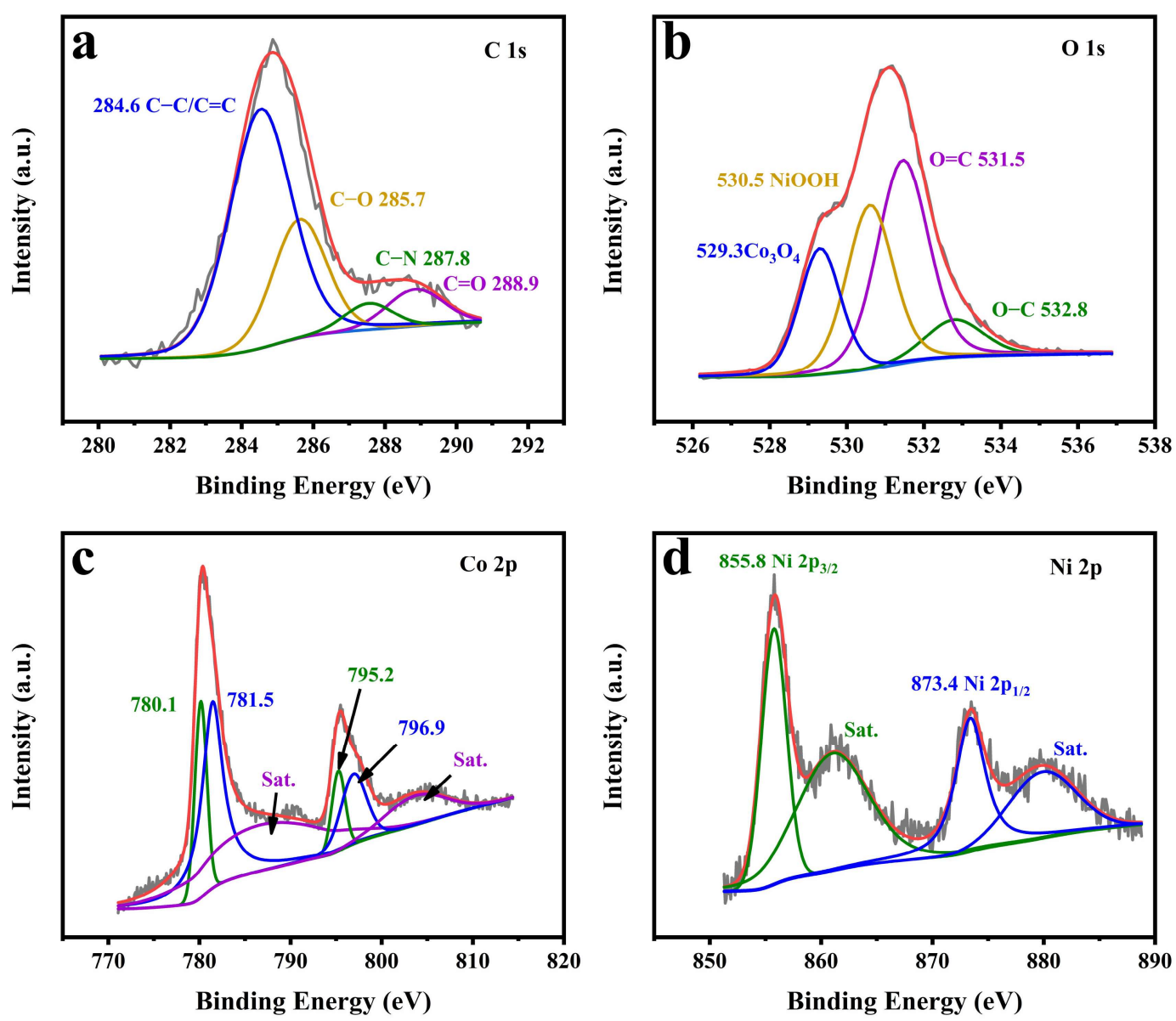

Fig. S11. High-resolution XPS spectra of $\mathrm{Co}_{0.4}(\mathrm{Hatz})_{0.1}\left(\mathrm{H}_{4} \mathrm{bta}\right)_{0.1} / \mathrm{NF}$ after OER stability test: (a) C 1s, (b) O 1s, (c) Co 2p and (d) Ni 2p. 
a

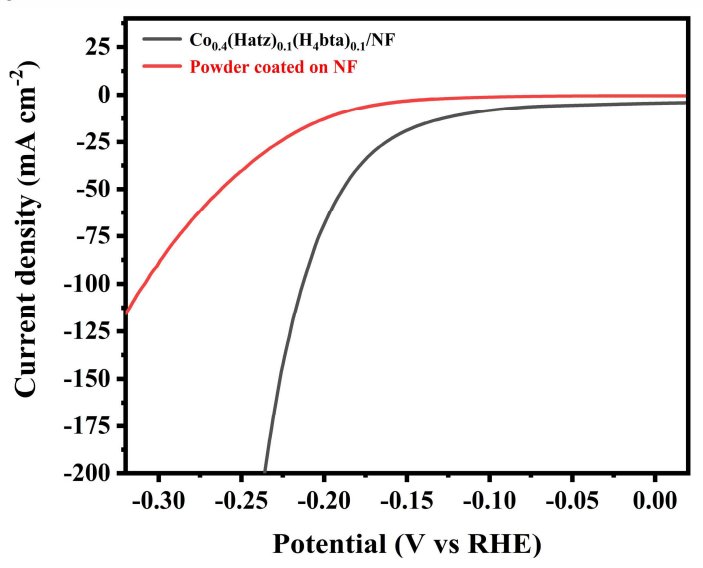

b

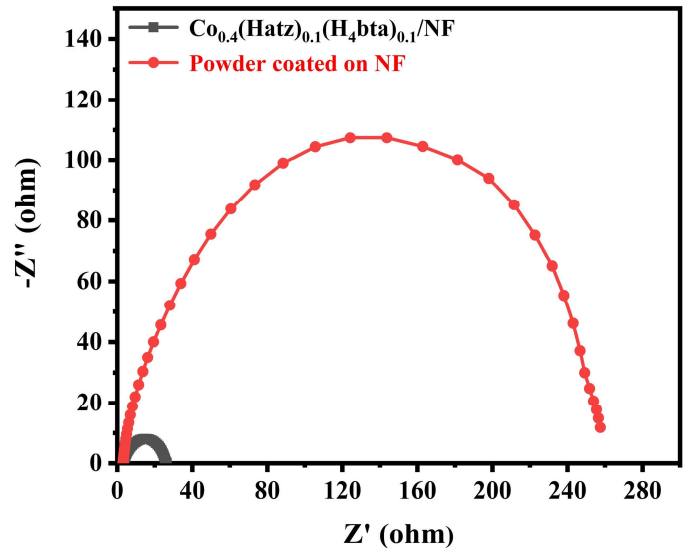

Fig. S12. Electrocatalytic HER performance of $\mathrm{Co}_{0.4}(\mathrm{Hatz})_{0.1}\left(\mathrm{H}_{4} \mathrm{bta}\right)_{0.1} / \mathrm{NF}$ and crystalline powder coated on the NF in 1.0 M KOH: (a) LSV polarization curves, (b) EIS of $\mathrm{Co}_{0.4}(\mathrm{Hatz})_{0.1}\left(\mathrm{H}_{4} \mathrm{bta}\right)_{0.1} / \mathrm{NF}$ and crystalline powder coated on the NF. 

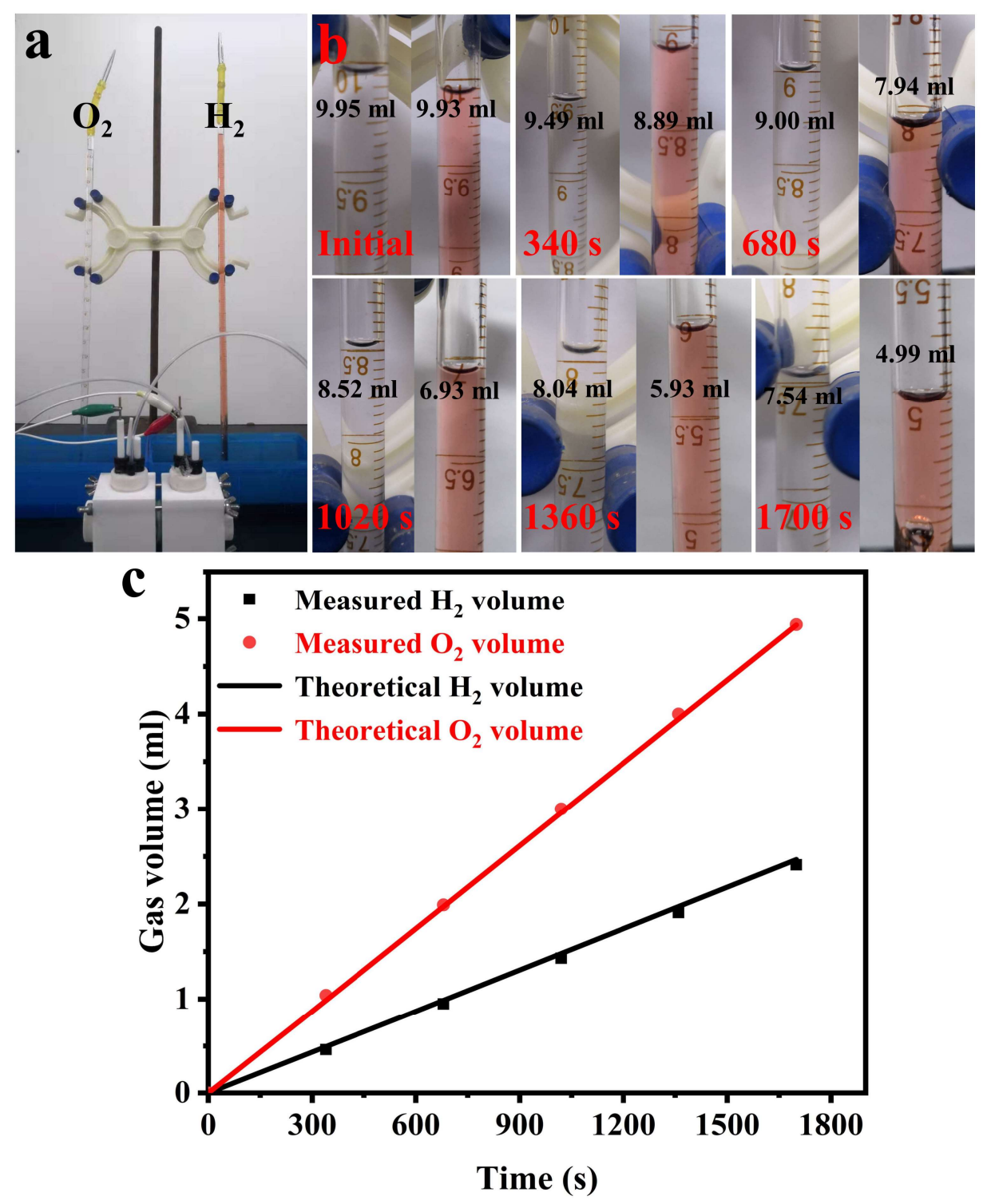

Fig. S13. (a) The digital photo of the experimental equipment, (b) The digital photo of $\mathrm{H}_{2}$ and $\mathrm{O}_{2}$ volume at different test time, (c) Volume of $\mathrm{H}_{2}$ and $\mathrm{O}_{2}$ as a function time at $0.025 \mathrm{~A}$.

The theoretical volume of $\mathrm{H}_{2}$ or $\mathrm{O}_{2}$ during overall water splitting can be calculated by Faraday law:

$$
\mathrm{V} / 22.4 \mathrm{~L} / \mathrm{mol}^{*} \mathrm{n} F=\mathrm{it}
$$

where, $\mathrm{V}$ is volume of $\mathrm{H}_{2}$ or $\mathrm{O}_{2}(\mathrm{~L}), \mathrm{n}$ is the electron number transferred to product one molecule gas, $F$ presents the faraday constant $\left(96485 \mathrm{C} \mathrm{mol}^{-1}\right) . \mathrm{i}$ is $0.025 \mathrm{~A}$ in procedure, $t$ represents test time (s). 
Table S1. The crystallographic date and structure refinement results of $\left\{\left[\mathrm{Co}_{2}(\mathrm{Hatz})(\mathrm{bta})\right] \cdot \mathrm{H}_{2} \mathrm{O}\right\}_{\mathrm{n}}$.

\begin{tabular}{lc}
\hline Complex & $\mathbf{1}$ \\
\hline Molecular formula & $\mathrm{C}_{12} \mathrm{H}_{6} \mathrm{Co}_{2} \mathrm{~N}_{4} \mathrm{O}_{9}$ \\
$\mathrm{M}$ & 468.07 \\
$T, \mathrm{~K}$ & $296(2) \mathrm{K}$ \\
Crystal system & Triclinic \\
Space group & $P-1$ \\
$a(\AA)$ & $7.4004(12)$ \\
$b(\AA)$ & $7.8438(13)$ \\
$c(\AA)$ & $13.606(2)$ \\
$\alpha\left(^{\circ}\right)$ & $77.159(3)$ \\
$\beta\left(^{\circ}\right)$ & $82.245(3)$ \\
$\gamma\left(^{\circ}\right)$ & $88.430(3)$ \\
$V\left(\AA^{3}\right)$ & $763.0(2)$ \\
$Z$ & 2 \\
$\rho(\mathrm{g}$ cm & -3 \\
$F(000)$ & 2.037 \\
Reflections & 464 \\
collected /unique & $4099 / 2866$ \\
{$[\mathrm{R}($ int $)]$} & 0.0252 \\
Goodness-of-fit on $\mathrm{F}^{2}$ & \\
Final $R$ indices $[I>2 \sigma(I)]$ & $R_{1}=0.0499$ \\
$R$ indices (all data) & $w R_{2}=0.1486$ \\
& $R_{1}=0.0758$ \\
\hline & $w R_{2}=0.1732$ \\
\hline
\end{tabular}


Table S2. The selected bond lengths and angles of $\left\{\left[\mathrm{Co}_{2}(\mathrm{Hatz})(\mathrm{bta})\right] \cdot \mathrm{H}_{2} \mathrm{O}\right\}_{\mathrm{n}}$.

\begin{tabular}{llll}
\hline Complex 1 & & & \\
\hline $\mathrm{Co}(1)-\mathrm{O}(3)$ & $2.026(4)$ & $\mathrm{N}(1)-\mathrm{Co}(1)-\mathrm{O}(1)$ & $177.8(2)$ \\
$\mathrm{Co}(1)-\mathrm{O}(5)$ & $2.042(4)$ & $\mathrm{O}(3)-\mathrm{Co}(1)-\mathrm{O}(4)$ & $148.26(18)$ \\
$\mathrm{Co}(1)-\mathrm{N}(1)$ & $2.070(5)$ & $\mathrm{O}(5)-\mathrm{Co}(1)-\mathrm{O}(4)$ & $92.28(18)$ \\
$\mathrm{Co}(1)-\mathrm{O}(1)$ & $2.082(4)$ & $\mathrm{N}(1)-\mathrm{Co}(1)-\mathrm{O}(4)$ & $92.63(18)$ \\
$\mathrm{Co}(1)-\mathrm{O}(4)$ & $2.099(4)$ & $\mathrm{O}(1)-\mathrm{Co}(1)-\mathrm{O}(4)$ & $88.40(16)$ \\
$\mathrm{Co}(2)-\mathrm{O}(2)$ & $1.983(4)$ & $\mathrm{O}(2)-\mathrm{Co}(2)-\mathrm{O}(6 \mathrm{~A})$ & $156.07(19)$ \\
$\mathrm{Co}(2)-\mathrm{O}(6 \mathrm{~A})$ & $2.025(4)$ & $\mathrm{O}(2)-\mathrm{Co}(2)-$ & $97.5(2)$ \\
& & $\mathrm{N}(2) \# 1$ & \\
$\mathrm{Co}(2)-\mathrm{N}(2) \# 1$ & $2.069(5)$ & $\mathrm{O}(6 \mathrm{~A})-\mathrm{Co}(2)-$ & $103.1(2)$ \\
$\mathrm{Co}(2)-\mathrm{O}(7)$ & $2.073(5)$ & $\mathrm{N}(2) \# 1$ & $\mathrm{O}(2)-\mathrm{Co}(2)-\mathrm{O}(7)$ \\
$\mathrm{Co}(2)-\mathrm{O}(4) \# 1$ & $2.182(4)$ & $\mathrm{O}(6 \mathrm{~A})-\mathrm{Co}(2)-\mathrm{O}(7)$ & $93.62(19)$ \\
$\mathrm{O}(3)-\mathrm{Co}(1)-\mathrm{O}(5)$ & $119.46(18)$ & $\mathrm{N}(2) \# 1-\mathrm{Co}(2)-$ & $94.3(2)$ \\
& & $\mathrm{O}(7)$ & $82.60(17)$ \\
$\mathrm{O}(3)-\mathrm{Co}(1)-\mathrm{N}(1)$ & $86.82(19)$ & $\mathrm{O}(2)-\mathrm{Co}(2)-$ & \\
& & $\mathrm{O}(4) \# 1$ & \\
$\mathrm{O}(5)-\mathrm{Co}(1)-\mathrm{N}(1)$ & $92.3(2)$ & $\mathrm{O}(6 \mathrm{~A})-\mathrm{Co}(2)-$ & \\
& & $\mathrm{O}(4) \# 1$ & \\
$\mathrm{O}(3)-\mathrm{Co}(1)-\mathrm{O}(1)$ & $91.28(17)$ & $\mathrm{N}(2) \# 1-\mathrm{Co}(2)-$ & \\
& & $\mathrm{O}(4) \# 1$ & $\mathrm{O}(7)-\mathrm{Co}(2)-$ \\
$\mathrm{O}(5)-\mathrm{Co}(1)-\mathrm{O}(1)$ & $89.60(18)$ & $\mathrm{O}(4) \# 1$ & \\
\hline
\end{tabular}


Table S3. The OER catalytic properties for different samples.

\begin{tabular}{|c|c|c|c|c|}
\hline samples & $\begin{array}{c}50 \mathrm{~mA} \mathrm{~cm}^{-2} \\
(\mathrm{mV})\end{array}$ & $\begin{array}{c}100 \mathrm{~mA} \mathrm{~cm}^{-} \\
2(\mathrm{mV})\end{array}$ & $\begin{array}{l}\text { Tafel slope } \\
\left(\mathrm{mV} \mathrm{dec}^{-1}\right)\end{array}$ & $\boldsymbol{R}_{\mathrm{ct}}(\Omega)$ \\
\hline $\mathrm{Co}_{0.1}(\mathrm{Hatz})_{0.1}\left(\mathrm{H}_{4} \mathrm{bta}\right)_{0.1} / \mathrm{NF}$ & 294 & 327 & 93 & 2.10 \\
\hline $\mathrm{Co}_{0.2}(\mathrm{Hatz})_{0.1}\left(\mathrm{H}_{4} \mathrm{bta}\right)_{0.1} / \mathrm{NF}$ & 332 & 361 & 96 & 4.78 \\
\hline $\mathrm{Co}_{0.4}(\mathrm{Hatz})_{0.1}\left(\mathrm{H}_{4} \mathrm{bta}\right)_{0.1} / \mathrm{NF}$ & 266 & 311 & 84 & 1.73 \\
\hline $\mathrm{Co}_{0.5}(\mathrm{Hatz})_{0.1}\left(\mathrm{H}_{4} \mathrm{bta}\right)_{0.1} / \mathrm{NF}$ & 361 & 393 & 109 & 8.51 \\
\hline $\mathrm{Co}(\mathrm{OH}) \mathrm{F} / \mathrm{NF}$ & 398 & 434 & 126 & - \\
\hline Nickel foam & 539 & 587 & 144 & 223 \\
\hline $\mathrm{IrO}_{2} / \mathrm{NF}$ & 302 & 333 & 72 & - \\
\hline
\end{tabular}


Table S4. EIS characterization of various electrodes. Values of the main parameters of equivalent circuit elements.

\begin{tabular}{cccc}
\hline Electrode & $\boldsymbol{R}_{\mathbf{s}}(\Omega)$ & $\boldsymbol{R}_{\mathbf{c}}(\Omega)$ & $\boldsymbol{R}_{\mathbf{c t}}(\Omega)$ \\
\hline $\mathrm{Co}_{0.1}(\mathrm{Hatz})_{0.1}\left(\mathrm{H}_{4} \mathrm{bta}\right)_{0.1} / \mathrm{NF}$ & $\sim 2.5 \Omega$ & 1.24 & 2.10 \\
$\mathrm{Co}_{0.2}(\mathrm{Hatz})_{0.1}\left(\mathrm{H}_{4} \mathrm{bta}\right)_{0.1} / \mathrm{NF}$ & $\sim 2.5 \Omega$ & 2.30 & 4.78 \\
$\mathrm{Co}_{0.4}(\mathrm{Hatz})_{0.1}\left(\mathrm{H}_{4} \mathrm{bta}\right)_{0.1} / \mathrm{NF}$ & $\sim 2.5 \Omega$ & 0.98 & 1.73 \\
$\mathrm{Co}_{0.5}(\mathrm{Hatz})_{0.1}\left(\mathrm{H}_{4} \mathrm{bta}\right)_{0.1} / \mathrm{NF}$ & $\sim 2.5 \Omega$ & 4.06 & 8.51 \\
\hline
\end{tabular}


Table S5. The HER catalytic properties for different samples.

\begin{tabular}{|c|c|c|c|c|}
\hline samples & $\begin{array}{c}10 \mathrm{~mA} \mathrm{~cm}^{-2} \\
(\mathrm{mV})\end{array}$ & $\begin{array}{c}50 \mathrm{~mA} \mathrm{~cm}^{-2} \\
(\mathrm{mV})\end{array}$ & $\begin{array}{l}\text { Tafel slope } \\
\left.(\mathrm{mV} \mathrm{dec})^{-1}\right)\end{array}$ & $\boldsymbol{R}_{\text {ct }}(\Omega)$ \\
\hline $\mathrm{Co}_{0.1}(\mathrm{Hatz})_{0.1}\left(\mathrm{H}_{4} \mathrm{bta}\right)_{0.1} / \mathrm{NF}$ & 129 & 194 & 87 & 31.4 \\
\hline $\mathrm{Co}_{0.2}(\mathrm{Hatz})_{0.1}\left(\mathrm{H}_{4} \mathrm{bta}\right)_{0.1} / \mathrm{NF}$ & 120 & 207 & 105 & 37.0 \\
\hline $\mathrm{Co}_{0.4}(\mathrm{Hatz})_{0.1}\left(\mathrm{H}_{4} \mathrm{bta}\right)_{0.1} / \mathrm{NF}$ & 115 & 189 & 78 & 23.4 \\
\hline $\mathrm{Co}_{0.5}(\mathrm{Hatz})_{0.1}\left(\mathrm{H}_{4} \mathrm{bta}\right)_{0.1} / \mathrm{NF}$ & 141 & 232 & 125 & 45.5 \\
\hline $\mathrm{Co}(\mathrm{OH}) \mathrm{F} / \mathrm{NF}$ & 202 & - & 206 & - \\
\hline Nickel foam & 146 & 226 & 116 & 60.0 \\
\hline $\mathrm{Pt} @ \mathrm{C} / \mathrm{NF}$ & 7 & 30 & 31 & - \\
\hline
\end{tabular}


Table S6. The comparison for overall water electrolysis activities for various catalysts.

\begin{tabular}{|c|c|c|c|c|}
\hline Materials & Supports & Electrolytes & $\begin{array}{c}E_{J}=10 \mathrm{~mA} \mathrm{~cm}^{-2} \\
\text { (V) }\end{array}$ & References \\
\hline Co-MOF/NF & Ni foam & $1 \mathrm{M} \mathrm{KOH}$ & 1.548 & This work \\
\hline $\mathrm{NiFe}-\mathrm{MOF} / \mathrm{NiSe}_{x} / \mathrm{NF}$ & Ni foam & $1 \mathrm{M} \mathrm{KOH}$ & 1.59 & 1 \\
\hline FexVy-PC/NF & Ni foam & $1 \mathrm{M} \mathrm{KOH}$ & 1.56 & 2 \\
\hline $\mathrm{FeNi}(\mathrm{BDC})(\mathrm{DMF}, \mathrm{F}) / \mathrm{NF}$ & Ni foam & $1 \mathrm{M} \mathrm{KOH}$ & 1.58 & 3 \\
\hline NFN-MOF/NF & $\mathrm{Ni}$ foam & $1 \mathrm{M} \mathrm{KOH}$ & 1.56 & 4 \\
\hline Co-Ni-Se/C/NF & Ni foam & $1 \mathrm{M} \mathrm{KOH}$ & 1.60 & 5 \\
\hline FeDy@MOF-Ni/CC & Carbon cloth & $1 \mathrm{M} \mathrm{KOH}$ & 1.57 & 6 \\
\hline $\mathrm{Co} / \mathrm{PNG}$ & $\begin{array}{c}\text { PNG } \\
\text { substrate }\end{array}$ & $1 \mathrm{M} \mathrm{KOH}$ & 1.61 & 7 \\
\hline
\end{tabular}




\section{References:}

(1) Xu, S.; Du, J.; Li, J.; Sun, L.; Li, F. Nickel-Selenide Templated Binary Metal-Organic Frameworks for Efficient Water Oxidation. J. Mater. Chem. A 2020, 8 (33), 16908-16912.

(2) Suo, N.; Han, X.; Chen, C.; He, X.; Dou, Z.; Lin, Z.; Cui, L.; Xiang, J.

Engineering Vanadium Phosphide by Iron Doping as Bifunctional

Electrocatalyst for Overall Water Splitting. Electrochim. Acta 2020, 333, 135531.

(3) Lin, H.-W.; Raja, D. S.; Chuah, X.-F.; Hsieh, C.-T.; Chen, Y.-A.; Lu, S.-Y. BiMetallic MOFs Possessing Hierarchical Synergistic Effects as High Performance Electrocatalysts for Overall Water Splitting at High Current Densities. Appl. Catal. B Environ. 2019, 258, 118023.

(4) Senthil Raja, D.; Chuah, X.-F.; Lu, S.-Y. In Situ Grown Bimetallic MOFBased Composite as Highly Efficient Bifunctional Electrocatalyst for Overall Water Splitting with Ultrastability at High Current Densities. Adv. Energy Mater. 2018, 8 (23), 1801065.

(5) Ming, F.; Liang, H.; Shi, H.; Xu, X.; Mei, G.; Wang, Z. MOF-Derived CoDoped Nickel Selenide/C Electrocatalysts Supported on Ni Foam for Overall Water Splitting. J. Mater. Chem. A 2016, 4 (39), 15148-15155.

(6) Wan, Z.; He, Q.; Chen, J.; Isimjan, T. T.; Wang, B.; Yang, X. DissolutionRegrowth of Hierarchical Fe-Dy Oxide Modulates the Electronic Structure of Nickel-Organic Frameworks as Highly Active and Stable Water Splitting Electrocatalysts. Chinese J. Catal. 2020, 41 (11), 1745-1753.

(7) Yang, G.; Liu, J.; Zhou, M.; Bai, J.; Bo, X. Fast and Facile Room-Temperature Synthesis of MOF-Derived Co Nanoparticle/Nitrogen-Doped Porous Graphene in Air Atmosphere for Overall Water Splitting. ACS Sustain. Chem. Eng. 2020, 8 (32), 11947-11955. 